\title{
A Retrospective Study Assessing the Effect of Diabetes on Mortality in Patients With COVID-19 at a Teaching Hospital in the United Kingdom
}

Fahad W. Ahmed ${ }^{1}$, Omar Z. Kirresh ${ }^{1}$, Alyss V. Robinson ${ }^{1}$, M S. Majeed ${ }^{2}$, Dominique Rouse ${ }^{1}$, Rumaisa Banatwalla ${ }^{1}$, Sathish Parthasarathy ${ }^{1}$, Catherine Sargent ${ }^{3}$, Clare Castledine ${ }^{4}$, Ali J. Chakera ${ }^{5}$

1. Diabetes and Endocrinology, Brighton and Sussex University Hospitals NHS Trust, Brighton, GBR 2. Diabetes and Endocrinology, Western Sussex Hospital NHS Foundation Trust, Worthing, GBR 3. Infectious Disease, Brighton and Sussex University Hospitals NHS Trust, Brighton, GBR 4. Medicine, Brighton and Sussex Medical School, Brighton, GBR 5. Diabetes and Endocrinology, Brighton and Sussex County Hospitals NHS Trust, Brighton, GBR

Corresponding author: Fahad W. Ahmed, fahadwali@yahoo.com

\section{Abstract}

\section{Aim}

The aim of the study was to compare the clinical characteristics and outcomes (mortality, intensive care admission, mechanical ventilation, and length of stay, LoS) of patients with and without diabetes with confirmed COVID-19.

\section{Methods}

This retrospective study evaluated clinical and laboratory variables in adult inpatients from Brighton and Sussex University Hospitals NHS Trust with laboratory-confirmed COVID-19 between March 10, 2020, and June 30, 2020. Univariate and multivariate analyses were performed to compare the outcomes of patients with and without diabetes.

\section{Results}

Over 457 patients were included in this study (140 with diabetes and 317 without diabetes), of which 143 (31.9\%) died. The median age was 80 years and were predominantly males (59.1\%). Baseline characteristics at the time of COVID-19 diagnosis demonstrated that the patients with diabetes were younger than those without diabetes $(\mathrm{p}=0.008)$. Mortality increased with age. There was no difference in adverse outcomes in those with and without diabetes. However, subgroup analysis of patients aged $\leqslant 60$ years demonstrated a significantly increased mortality in those with diabetes $(\mathrm{p}=0.016)$. Patients with diabetes had an increased length-of-stay compared to those without diabetes, which was more evident in those aged $\leqslant 60$ years.

\section{Conclusion}

Review began 03/08/2021 Review ended 03/13/2021 Published 03/15/2021

\section{() Copyright 2021}

Ahmed et al. This is an open access article distributed under the terms of the Creative Commons Attribution License CC-BY 4.0., which permits unrestricted use, distribution, and reproduction in any medium, provided the original author and source are credited.
Age is the most important predictor of mortality. Patients with diabetes did not have increased mortality from COVID-19, which is likely due to their younger age in our cohort. More patients with diabetes stayed in the hospital longer than seven days than those without diabetes.

Categories: Endocrinology/Diabetes/Metabolism, Infectious Disease

Keywords: coronavirus disease 2019, covid-19, diabetes mellitus, united kingdom, mortality

\section{Introduction}

The world is presently undergoing a significant health crisis due to coronavirus disease 2019 (COVID-19). COVID-19 is caused by severe acute respiratory syndrome coronavirus 2 (SARS-CoV-2). The World Health Organisation declared COVID-19 as a pandemic on March 11, 2020 [1]. Two days later, Europe was declared as an epicenter of the pandemic [2]. On October 4, 2020, there were approximately 35 million cases of COVID-19 resulting in over one million deaths worldwide [3].

Several studies have demonstrated a higher proportion of patients with diabetes are admitted to hospital due to COVID-19 [4,5]. Data from the United States identified that out of 1122 patients hospitalized due to COVID-19, 40.2\% ( $\mathrm{n}=451)$ had diabetes [4]. One retrospective study from the United Kingdom (UK) observed that $37.5 \%$ of patients admitted with COVID-19 had co-existing diabetes [5]. Multiple retrospective studies and systematic reviews have demonstrated that diabetes and intercurrent COVID-19 are associated with adverse outcomes, including increased mortality [4,6-8].

This study reports the outcomes of patients admitted with COVID-19 at Brighton Sussex University Hospitals NHS Trust (BSUH), UK. This study evaluates the impact of diabetes on mortality, intensive care 


\section{Materials And Methods}

\section{Study design}

This retrospective cross-sectional observational study was conducted in BSUH, which comprises of two hospitals: Royal Sussex County Hospital and Princess Royal Hospital. BSUH serves a population of approximately 500,000 people. All adult patients ( 18 -years) hospitalized between March 10, 2020, and June 30, 2020, with laboratory-confirmed COVID-19 [a reverse-transcriptase-polymerase-chain-reaction (RTPCR) SARS-CoV-2 assay of a nasopharyngeal swab], were included in the analysis. Patients were excluded from the analysis if they were discharged from the emergency department, transferred to another center (predominantly occurring in the early stages of the COVID-19 pandemic in the UK), or there was insufficient clinical information. Ethics approval was not required as the data were collected anonymously as part of routine healthcare delivery and service improvement analysis.

\section{Data collection}

Clinical and laboratory data were extracted from electronic hospital records. The clinical information collected were age, biological sex, ethnicity, socio-economic status, pre-existing diabetes or no pre-existing diabetes, type of diabetes (type 1 diabetes mellitus or type 2 diabetes mellitus), co-morbidities [chronic obstructive pulmonary disease (COPD), asthma, heart disease, hypertension, malignancy], length-of-stay, admission to intensive care unit (ITU), requiring mechanical ventilation and mortality. The laboratory parameters collected were glycated hemoglobin (HbA1c), estimated glomerular filtration rate (eGFR), and admission venous blood gas (VBG) glucose. Ward-based capillary glucose readings were reviewed to evaluate inpatient glycaemic control for patients with diabetes.

Diabetes was defined as an $\mathrm{HbA} 1 \mathrm{c} \geqslant 6.5 \%$ ( $\geqslant 48 \mathrm{mmol} / \mathrm{mol}$ ) on admission or an established diagnosis of diabetes prior to hospitalization. Socio-economic status was based on English Indices of Multiple Deprivation for the postcode (2019) [9]. Index of multiple deprivations provides an official measure of deprivation of a local area across England and is based on different factors, e.g., income, employment, education, health and disability, crime, living environment, and housing barriers [9]. The area with an index of multiple deprivation score of 1 is the most deprived area whereas the area with an index of multiple deprivations of 10 is the least deprived area [9]. Diabetic ketoacidosis (DKA) and hyperosmolar hyperglycaemic state (HHS) were defined as per the Joint British Diabetes Societies (JBDS) guidance [10,11]. Hyperglycaemia on admission was described as a blood glucose level $>11 \mathrm{mmol} / \mathrm{l}$ at the time of admission on VBG. Hyperglycaemia during admission was analyzed in two ways (1) average blood glucose level >11 mmol/1 during admission (capillary blood glucose) and (2) two blood glucose readings $>11 \mathrm{mmol} / \mathrm{l}$ within a 24 -hour period during admission. Hypoglycemia at presentation was defined as blood glucose $<4 \mathrm{mmol} / \mathrm{L}$.

\section{Statistical analysis}

Categorical variables were reported as percentages. Continuous variables were reported as a median and inter-quartile range (IQR) as they were not normally distributed. Variables were compared between patients with diabetes and without diabetes. Furthermore, we compared ITU admissions, mechanical ventilation, and length of stay (LoS) between patients based on diabetes status and age $\leqslant 60$ and $>60$ years. We also compared age between ethnic groups.

In patients with diabetes, individual variables were assessed according to mortality outcomes. For continuous variables, the Mann-Whitney U test was used for non-normally distributed variables, whereas Pearson's chi-squared test $\left(\chi^{2}\right)$ was used for categorical variables.

Logistic regression analysis was undertaken to calculate the odds for all-cause 28-day mortality in both the whole cohort and in the diabetes subset. Variables with a p-value $<0.05$ in the univariate analysis and those thought to be clinically related to the associations examined were included in the multivariate analysis.

All-cause mortality was calculated as time, in days, between the first positive RT-PCR SARS-CoV-2 swab date and the date of death within 28 days of a positive COVID-19 test. Follow-up is defined from the date of positive RT-PCR SARS-CoV-2 swab to death or within 28 days of a positive COVID-19 test (whichever comes first). This is based on the Public Health England criteria used to calculate COVID-19 daily death [12]. Survival analysis was undertaken using the Kaplan-Meier survival curve based on (1) diabetes status (all ages included), and ( 2 ) diabetes and age $\leqslant 60$ years and $>60$ years. A p-value of $<0.05$ was considered statistically significant. The analysis was performed with SPSS software (version 23.0, IBM Corp., Armonk, NY).

\section{Results}

\section{Overall results}

During the study period, 550 patients were identified to have tested positive for SARS-CoV-2. After preprotocol exclusions, 457 patients were included in the analysis (Figure 1). Baseline characteristics and 


\section{Cureus}

laboratory findings of all patients and those with and without diabetes are summarised in Table 1. There was a greater proportion of males (59.1\%) than females, and the median age was 80 years. The median LoS was 14 days (6-26). Out of 457 patients, 140 (30.6\%) had diabetes and 143 (31.9\%) patients with COVID-19 died. Patients from a Black, Asian, and minority ethnic (BAME) background were significantly younger than patients from a White background [median (IQR); BAME: 60.5 (46-72) years vs 81 (69.50-87) years: $\mathrm{p}<0.0001]$.

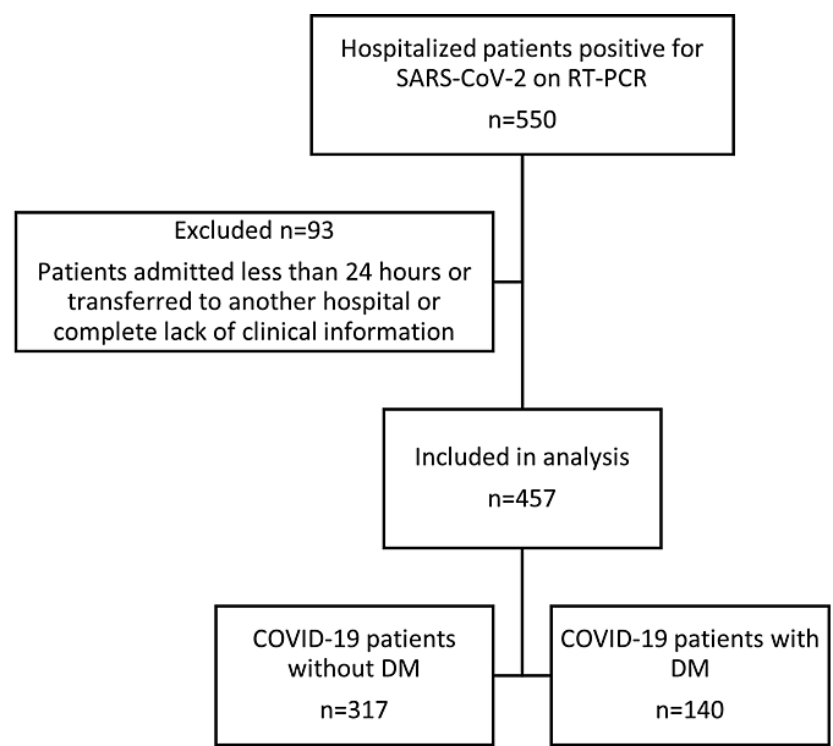

FIGURE 1: Flow diagram of patients included in the study.

COVID-19: coronavirus, SARS-COV-2: severe acute respiratory syndrome coronavirus-2, RT-PCR: real-time reverse transcription-polymerase chain reaction, $n$ : number. 


\begin{tabular}{|c|c|c|c|c|c|c|c|}
\hline & $\mathbf{n}$ & All & $\mathbf{n}$ & Non-diabetes & $\mathbf{n}$ & Diabetes & p-Value \\
\hline \multicolumn{8}{|l|}{ Demographics } \\
\hline Male sex (n, \%) & 457 & $270(59.1 \%)$ & 317 & 185 (58.4\%) & 140 & $85(60.7 \%)$ & 0.68 \\
\hline Age (median, IQR) & 457 & $80(67-87)$ & 317 & $81(68-87)$ & 140 & $76(64-85)$ & 0.008 \\
\hline Ethnicity (n, \%) & 457 & & 317 & & 140 & & \\
\hline White & & $389(85.1 \%)$ & & $279(88 \%)$ & & 110 (78.6\%) & \\
\hline BAME & & $46(10.1 \%)$ & & $21(6.6 \%)$ & & $25(17.9 \%)$ & 0.001 \\
\hline Unknown & & $22(4.8 \%)$ & & $17(5.4 \%)$ & & $5(3.6 \%)$ & \\
\hline \multicolumn{8}{|l|}{ Co-morbidities (n, \%) } \\
\hline Asthma & 449 & $45(9.8 \%)$ & 311 & $25(8 \%)$ & 138 & $20(14.5 \%)$ & 0.04 \\
\hline COPD & 449 & $59(12.9 \%)$ & 311 & $34(10.9 \%)$ & 138 & $25(18.1 \%)$ & $<0.05$ \\
\hline Heart disease & 449 & $172(37.6 \%)$ & 311 & $105(33.8 \%)$ & 138 & $67(48.6 \%)$ & 0.003 \\
\hline Hypertension & 449 & $187(40.9 \%)$ & 311 & $109(35 \%)$ & 138 & $78(56.5 \%)$ & $<0.0001$ \\
\hline Cancer & 448 & $98(21.4 \%)$ & 311 & $66(21.2 \%)$ & 137 & $32(23.4 \%)$ & 0.62 \\
\hline \multicolumn{8}{|l|}{ Laboratory findings } \\
\hline HbA1c (median, IQR) & 358 & $41(37-49)$ & 244 & $38(35-41)$ & 134 & $55.5(46-70.25)$ & 0.0001 \\
\hline Venous glucose (median, IQR) & 413 & $6.7(5.8-8.75)$ & 288 & $6.35(5.57-7.2)$ & 125 & $9.2(6.95-12.2)$ & $<0.0001$ \\
\hline eGFR (n, \%) & 456 & & & & & & \\
\hline$\geq 60$ & & $255(55.9 \%)$ & & $192(60.6 \%)$ & & $63(45.3 \%)$ & \multirow{3}{*}{0.03} \\
\hline$<60$ & & 201 (44.1\%) & & $125(39.4 \%)$ & & $76(54.7 \%)$ & \\
\hline Index of multiple deprivation decile (n, \%) & 457 & & 317 & & 140 & & \\
\hline $1-3$ & & 78 (17.1\%) & & $53(16.7 \%)$ & & $25(17.9 \%)$ & \\
\hline 4-7 & & $191(41.8 \%)$ & & $130(41 \%)$ & & $61(43.6 \%)$ & 0.76 \\
\hline $8-10$ & & $188(41.1 \%)$ & & $134(42.3 \%)$ & & $54(38.6 \%)$ & \\
\hline \multicolumn{8}{|l|}{ Outcomes (n, \%) } \\
\hline Death & 457 & $143(31.9 \%)$ & 317 & $101(31.9 \%)$ & 140 & $42(30.0 \%)$ & 0.74 \\
\hline ITU admission & 457 & $52(11.4 \%)$ & 317 & $34(10.7 \%)$ & 140 & $18(12.9 \%)$ & 0.53 \\
\hline Mechanical ventilation & 457 & $18(3.9 \%)$ & 317 & 12 (3.8\%) & 140 & $6(4.3 \%)$ & 0.80 \\
\hline LoS (median, IQR) & 457 & $14(6-26)$ & 317 & $13(5-25)$ & 140 & $14.5(8.25-26.75)$ & 0.057 \\
\hline \multicolumn{8}{|l|}{$\operatorname{LoS}(\mathrm{n}, \%)$} \\
\hline$<7$ days & & 127 (27.8\%) & & $100(31.6 \%)$ & & 27 (19.3\%) & \multirow{3}{*}{0.019} \\
\hline $7-14$ days & & $116(25.4 \%)$ & & $73(23 \%)$ & & 43 (30.7\%) & \\
\hline$>14$ days & & $214(46.8 \%)$ & & $144(45.4 \%)$ & & $70(50 \%)$ & \\
\hline
\end{tabular}

TABLE 1: Baseline characteristics and outcomes for all hospitalized COVID-19 patients with and without diabetes.

n: number of patients, \%: percentage, BAME: Black, Asian and Minority Ethnic, COPD: chronic obstructive pulmonary disease, eGFR: estimated glomerular filtration rate, ITU: intensive care, IQR: interquartile range, LoS: length-of-stay. 


\section{Cureus}

Patients with diabetes were significantly younger and with more co-morbidities than those without diabetes (Table 1). There was a significantly greater proportion of BAME backgrounds in those with diabetes (17.9\%) compared to those without diabetes $(6.6 \% ; \mathrm{p}<0.001)$. Patients from a BAME background in our cohort were significantly younger than those from a White background in both diabetes and non-diabetes groups [median (IQR); diabetes: BAME - 66 (49-79) years vs White - 78 (67-85.25) years: $\mathrm{p}=0.017$; non-diabetes: BAME - 55 (43.50-67.50) years vs White - $81(59.50-77)$ years: $\mathrm{p}<0.0001]$.

There was no significant difference in mortality outcomes and median LoS in patients with and without diabetes. However, the distribution of LoS demonstrated more patients without diabetes were discharged within a week of admission.

Admission blood glucose was significantly higher in those with diabetes $(9.2 \mathrm{mmol} / \mathrm{L})$ compared to those without diabetes $(6.35 \mathrm{mmol} / \mathrm{L} ; \mathrm{p}<0.0001)$. There was a significant difference in the number of patients with an eGFR<60 in patients with diabetes than those without diabetes ( $54.7 \%$ versus $39.4 \%$, p=0.03).

The number of patients requiring ITU admission and mechanical ventilation and length-of-stay outcomes of COVID-19 patients based on age ( $\leqslant 60$ years compared to $>60$ years) and diabetes status are provided in Table 2. There was no significant difference in age between diabetes and non-diabetes groups. In patients aged $\leqslant 60$ years, those with diabetes had a significantly longer LoS than those without diabetes [median (IQR); Diabetes: 11 (8-16.75) days vs non-diabetes: 5 (3-12.25) days: $p=0.002]$; 53.8\% with diabetes had an LoS of 714 days, whilst $12.5 \%$ without diabetes had an LoS of 7-14 days. There was no significant difference between ITU admissions and mechanical ventilation in patients $\leqslant 60$ years with diabetes and those without diabetes.

\begin{tabular}{|c|c|c|c|}
\hline & Non-diabetes & Diabetes & p-Value \\
\hline \multicolumn{4}{|l|}{ Number of patients (n, \%) } \\
\hline$\leq 60$ years & 48 (15.1\%) & 26 (18.6\%) & 0.41 \\
\hline$>60$ years & 269 (84.9\%) & $114(81.4 \%)$ & \\
\hline \multicolumn{4}{|l|}{ ITU admission (n, \%) } \\
\hline Age $\leq 60$ years & $8(16.7 \%)$ & $7(26.9 \%)$ & 0.37 \\
\hline Age $>60$ years & 26 (9.7\%) & $11(9.6 \%)$ & 0.99 \\
\hline Mechanical ventilation (n, \%) & $12(3.8 \%)$ & $6(4.3 \%)$ & 0.80 \\
\hline Age $\leq 60$ years & $3(6.3 \%)$ & $1(3.8 \%)$ & 0.99 \\
\hline Age $>60$ years & $9(3.3 \%)$ & $5(4.4 \%)$ & 0.77 \\
\hline \multicolumn{4}{|l|}{ LoS (n, \%) } \\
\hline \multicolumn{4}{|l|}{ Age $\leq 60$ years } \\
\hline$<7$ days & $31(64.6 \%)$ & $4(15.4 \%)$ & $<0.0001$ \\
\hline 7-14 days & $6(12.5 \%)$ & 14 (53.8\%) & \\
\hline$>14$ days & $11(22.9 \%)$ & $8(30.8 \%)$ & \\
\hline \multicolumn{4}{|l|}{ Age $>60$ years } \\
\hline$<7$ days & $69(25.9 \%)$ & $23(20.2 \%)$ & 0.50 \\
\hline 7-14 days & $67(24.9 \%)$ & 29 (25.4\%) & \\
\hline >14 days & 133 (49.4\%) & 62 (54.4\%) & \\
\hline
\end{tabular}

TABLE 2: ITU admission, mechanical ventilation, and length outcomes of COVID-19 patients based on age ( $\leq 60$-years-old compared to $>60$-years-old) and diabetes status hospitalized at Brighton and Sussex University Hospitals.

ITU: intensive care, n: number, \%: percentage, LoS: length of stay. 
26) days: $p=0.24]$, ITU admissions, and mechanical ventilation in patients aged $>60$ years in those with and without diabetes.

\section{Characteristics in COVID-19 patients with diabetes and mortality outcome}

There was no significant difference in the mortality outcomes of COVID-19 patients with diabetes (Table 3) in relation to biological sex, age, ethnicity, type of diabetes, index of deprivation decile, HbA1c, control of diabetes (VBG glucose, average glucose, hyperglycemia at presentation, hyperglycaemic emergency, hypoglycemia, and the number of capillary blood glucose readings) co-morbidities, and eGFR. Average capillary blood glucose was [median (IQR) 8.6 (7.1-10.33) $\mathrm{mmol} / \mathrm{L}]$.

\begin{tabular}{|c|c|c|c|c|c|}
\hline & $\mathbf{n}$ & Not alive & $\mathbf{n}$ & alive & p-Value \\
\hline \multicolumn{6}{|l|}{ Demographics } \\
\hline Male sex (n, \%) & 42 & $28(66.7 \%)$ & 98 & $57(58.2 \%)$ & 0.45 \\
\hline Age (median, IQR) & 42 & $78(70-85)$ & 98 & 72.5 (61.75-85.25) & 0.19 \\
\hline \multicolumn{6}{|l|}{ Age (n, \%) } \\
\hline$\leq 60$ years & & $3(7.1 \%)$ & & $23(23.5 \%)$ & \multirow{2}{*}{0.03} \\
\hline$>60$ years & & 39 (92.9\%) & & 75 (76.5\%) & \\
\hline Ethnicity (n, \%) & 42 & & 98 & & \\
\hline White & & 35 (83.3\%) & & 75 (76.5\%) & \multirow{3}{*}{0.66} \\
\hline BAME & & $6(14.3 \%)$ & & 19 (19.4\%) & \\
\hline Unknown & & $1(2.4 \%)$ & & $4(4.1 \%)$ & \\
\hline \multicolumn{6}{|l|}{ Co-morbidities (n, \%) } \\
\hline Asthma & 40 & $2(5 \%)$ & 98 & 18 (18.4\%) & 0.06 \\
\hline COPD & 40 & $10(25 \%)$ & 98 & 15 (15.3\%) & 0.22 \\
\hline Heart disease & 40 & $20(50 \%)$ & 98 & 47 (48\%) & 0.85 \\
\hline Hypertension & 40 & $21(52.5 \%)$ & 98 & 57 (58.2\%) & 0.57 \\
\hline Cancer & 40 & $11(27.5 \%)$ & 97 & $21(21.6 \%)$ & 0.51 \\
\hline \multicolumn{6}{|l|}{ Glycemic parameters } \\
\hline HbA1c (median, IQR) & 39 & $57(48-68)$ & 95 & $53(45-72)$ & 0.51 \\
\hline $\mathrm{HbA} 1 \leq 69$ mmol/mol* $(\mathrm{n}, \%)$ & & $31(79.5 \%)$ & & $68(71.6 \%)$ & \multirow{2}{*}{0.39} \\
\hline $\mathrm{HbA} 1 \mathrm{c}>69 \mathrm{mmol} / \mathrm{mol}^{\wedge}$ (n, \%) & & $8(20.5 \%)$ & & $27(28.4 \%)$ & \\
\hline Venous glucose (median, IQR) & & $8.6(6.7-12.2)$ & & $9.5(7.2-12.4)$ & 0.44 \\
\hline Hyperglycemia > 11mmol/L, two readings per day (n. \%) & 40 & $24(61.5 \%)$ & 94 & $53(57 \%)$ & 0.70 \\
\hline Average glucose, mmol/L (median, IQR) & 40 & 8.55 (7.1-10.23) & 94 & $8.6(7.2-10.9)$ & 0.57 \\
\hline Hyperglycemia with average reading > 11 mmol/L (n, \%) & 40 & 9 (22.5\%) & 94 & $18(19.1 \%)$ & 0.65 \\
\hline Point of care readings (median, IQR) & 42 & $3.4(2.4-4.4)$ & 98 & $3.4(1.9-4.4)$ & 0.58 \\
\hline Point of care readings $(\mathrm{n}, \%)$ & 42 & & 98 & & \\
\hline$\leq 2$ & & $10(24.4 \%)$ & & $30(30.6 \%)$ & \multirow{2}{*}{0.54} \\
\hline$>2$ & & $31(75.6 \%)$ & & $68(69.4 \%)$ & \\
\hline Hyperglycemia on admission (n, \%) & 40 & $11(27.5 \%)$ & 85 & $28(32.9 \%)$ & 0.68 \\
\hline DKA or/and HHS, n (\%) & 42 & $2(4.8 \%)$ & 98 & $3(3.1 \%)$ & 0.64 \\
\hline Hypoglycemia on admission (n, \%) & 42 & $1(2.4 \%)$ & 98 & $1(1 \%)$ & 0.51 \\
\hline
\end{tabular}




\section{Cureus}

Laboratory findings

eGFR (n, \%)

$<60$

$\geq 60$

Index of multiple deprivation decile (n, \%)

1-3

4-7

8-10
42

$15(35.7 \%)$

$27(64.3 \%)$

42

$9(21.4 \%)$

$20(47.6 \%)$

$13(31 \%)$
97

$48(49.5 \%)$

$49(50.5 \%)$

98

$16(16.3 \%)$

$41(41.8 \%)$

0.46

TABLE 3: Clinical characteristics based on mortality outcomes for hospitalized patients with COVID-19 and diabetes.

n: number, \%: percentage, BAME: Black Asian Minority Ethnicity, LoS: length-of-stay, COPD: chronic obstructive pulmonary disease, HTN: hypertension, ITU: intensive care unit, IQR: intra-quartile, eGFR: estimated glomerular filtration rate, DKA: diabetic ketoacidosis, HHS: hyperosmolar hyperglycemic state.

${ }^{*} \leq 8.5 \%, \wedge>8.5 \%$.

\section{Risk factors associated with mortality}

Table 4 describes univariate and multivariate logistic regression analyses of the risk factors for mortality in all patients with COVID-19 hospitalized in BSUH, UK. In the multivariate analysis of all the patients with COVID-19, age $>60$ years and an eGFR $<60$ were independent risk factors associated with increased mortality. 


\begin{tabular}{|c|c|c|c|c|c|c|}
\hline & \multicolumn{4}{|c|}{ All participants } & \multirow{2}{*}{\multicolumn{2}{|c|}{$\begin{array}{l}\text { Diabetes } \\
\text { Univariate }\end{array}$}} \\
\hline & \multicolumn{2}{|l|}{ Univariate } & \multicolumn{2}{|c|}{ Multivariate } & & \\
\hline & $\mathrm{p}$-Value & OR; Cl 95\% & $\mathrm{p}$-Value & OR; Cl 95\% & $\mathrm{p}$-Value & OR; $\mathrm{Cl}$ 95\% \\
\hline Age $>60$ years ${ }^{\star *}$ & $<0.0001^{*}$ & $13.64(4.22-44.10)$ & 0.001 & $12.78(3.01-54.22)$ & 0.032 & $3.99(1.13-14.11)$ \\
\hline Male & 0.052 & $1.504(0.99-2.27)$ & ND & ND & 0.342 & $1.44(0.68-3.07)$ \\
\hline Diabetes & 0.392 & $0.92(0.60-1.41)$ & ND & ND & ND & ND \\
\hline Asthma & 0.059 & $0.47(0.21-1.03)$ & ND & ND & 0.06 & $0.23(0.05-1.06)$ \\
\hline COPD & $0.006^{*}$ & $2.18(1.25-3.80)$ & 0.058 & $1.75(0.98-3.12)$ & 0.18 & $1.84(0.75-4.55)$ \\
\hline Heart disease & $0.037^{*}$ & $1.55(1.03-2.33)$ & 0.95 & $1.01(0.65-1.58)$ & 0.83 & $1.09(0.52-2.26)$ \\
\hline Hypertension & 0.732 & $0.93(0.62-1.40)$ & ND & ND & 0.54 & $0.80(0.38-1.67)$ \\
\hline Cancer & $0.026^{*}$ & $1.70(1.07-2.71)$ & 0.16 & $1.42(0.87-2.33)$ & 0.46 & $1.37(0.60-3.20)$ \\
\hline Risk Factors (1 or more) & 0.273 & $0.91(0.78-1.07)$ & ND & ND & $0.23^{+}$ & $1.45(0.79-2.64)$ \\
\hline eGFR $<60 \mathrm{~mL} / \mathrm{min} / 1.73 \mathrm{~m}^{2}$ & $<0.001^{\star}$ & $2.49(1.678-3.73)$ & 0.001 & $2.06(1.33-3.20)$ & 0.14 & $1.76(0.84-3.72)$ \\
\hline Hyperglycemia on presentation & 0.820 & $0.93(0.49-1.77)$ & ND & ND & 0.54 & $0.77(0.34-1.77)$ \\
\hline BAME & $0.036^{*}$ & $0.43(0.20-0.95)$ & 0.24 & $0.55(0.21-1.47)$ & 0.45 & $0.68(0.25-1.84)$ \\
\hline Type 2 diabetes ${ }^{\wedge}$ & ND & ND & ND & ND & 0.62 & $0.63(0.10-3.93)$ \\
\hline Hyperglycemic emergencies & ND & ND & ND & ND & 0.62 & $1.58(0.26-9.84)$ \\
\hline Hyperglycemia (two readings $>11 \mathrm{mmol} / \mathrm{L}$ ) & ND & ND & ND & ND & 0.63 & $1.21(0.56-2.59)$ \\
\hline Average glucose $>11 \mathrm{mmol} / \mathrm{L}$ & ND & ND & ND & ND & 0.66 & $1.23(0.50-3.02)$ \\
\hline Number of blood glucose reading per day & ND & ND & ND & ND & 0.46 & $1.37(0.60-3.14)$ \\
\hline $\mathrm{HbA} 1 \mathrm{c}>69^{\wedge} \mathrm{mmol} / \mathrm{mol}$ & ND & ND & ND & ND & 0.35 & $0.65(0.26-1.59)$ \\
\hline
\end{tabular}

TABLE 4: Logistic regression analysis for evaluating the risk factors for mortality in all patients with COVID-19 hospitalized in BSUH, United Kingdom.

$\mathrm{P}<0.05$ was considered significant.

*Used for multivariate analysis, ${ }^{\star *}$ compared to $\leq 60$ years of age, ${ }^{\star * \star}$ include diabetes, asthma, COPD, heart disease, hypertension and cancer, ${ }^{\wedge} \mathrm{HbA} 1 \mathrm{c}>8.5 \%,{ }^{\wedge}$ compared to type 1 diabetes, +diabetes excluded from risk factor (included Asthma, COPD, heart disease, hypertension and cancer), COPD: chronic obstructive pulmonary disease, BAME: Black Asian Minority Ethnicity, eGFR: estimated glomerular filtration rate, ND: not done.

Univariate analysis (Table 4 ) of patients with diabetes found that age $>60$ was the only risk factor; therefore, multivariate analysis was not performed.

\section{Survival analysis}

Kaplan-Meier survival curves demonstrated no difference in survival in hospitalized COVID-19 patients with diabetes compared to those without diabetes (Figure 2). However, patients aged $\leqslant 60$ years with diabetes $(\mathrm{n}=3)$ were significantly less likely $(\mathrm{p}=0.016)$ to survive than those without diabetes $(\mathrm{n}=0$; Figure 3$)$. Out of three, two patients were from BAME background. No significant difference was demonstrated in survival in the patients aged $>60$ years with or without diabetes (Figure 4). 


\section{Cureus}

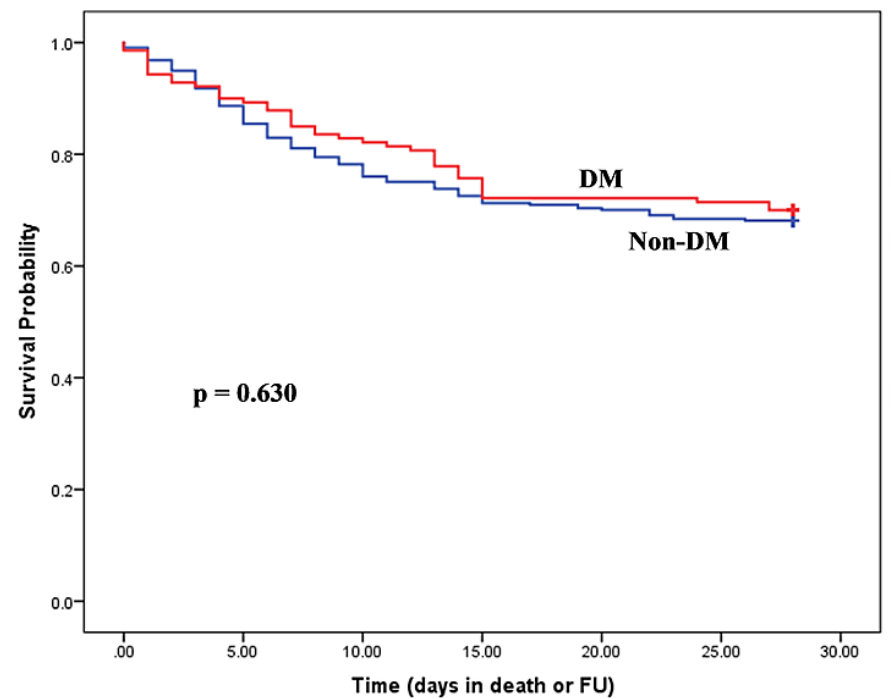

FIGURE 2: Kaplan-Meier estimates of survival for COVID-19 patients with and without diabetes mellitus.

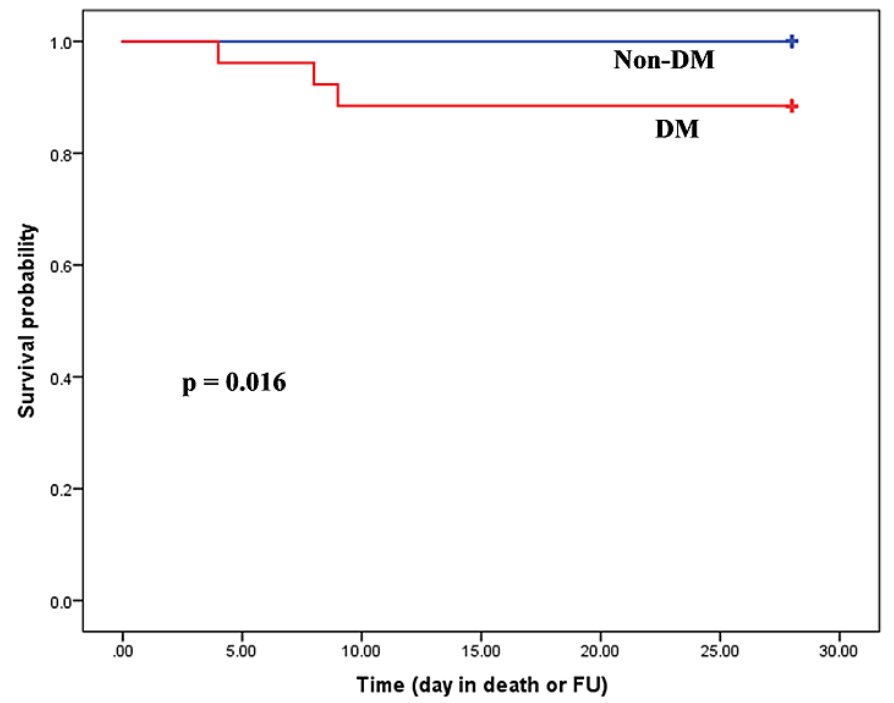

FIGURE 3: Kaplan-Meier estimates of survival for COVID-19 patients with and without diabetes mellitus aged $\leq 60$ years. 


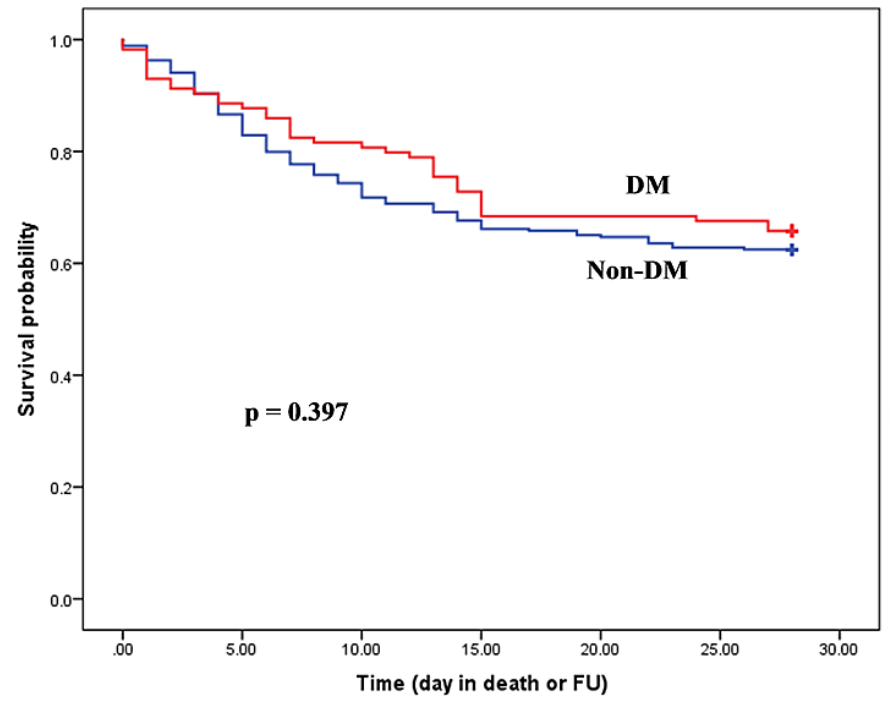

FIGURE 4: Kaplan-Meier estimates of survival for COVID-19 patients with and without diabetes mellitus aged $>60$ years.

\section{Discussion}

This retrospective study described clinical characteristics of patients with and without diabetes, and COVID-19 admitted to a tertiary care hospital in the United Kingdom. Around a third (140 out of 457) of the patients with COVID-19 had diabetes. This study demonstrated that patients with diabetes did not have an increased risk of death, ITU admission, or mechanical ventilation. This was despite patients with diabetes having more co-morbidities and a larger proportion of BAME background. This lack of difference is likely due to the age difference between diabetes and non-diabetes groups. Subgroup analysis revealed significantly increased mortality in patients with diabetes who were aged $\leqslant 60$ years compared to those without diabetes, though the numbers involved in this subgroup analysis included very few deaths.

\section{Characteristics of all patients admitted with COVID-19}

This study showed that most of the patients admitted were male (59.1\%) and elderly (median age of 80 years). There were fivefold more patients aged $>60$ years compared to those aged $\leqslant 60$ years. Furthermore, age $>60$ years was associated with increased mortality. These findings are consistent with current worldwide evidence [13], including the UK [5,14]. A UK study confirmed a 20-fold increased risk of COVID-19-related mortality in peoples aged $>80$ years compared to those $<60$ years [14]. The cohort in our study was significantly older (median 80 years, mean 75 years) when compared to evidence emerging from China (60 years) [15] and Europe (70 years) $[5,16]$. While male sex has been associated with increased mortality from COVID-19 [14,17], we did not directly observe this in our study. However, a greater proportion of hospitalized patients were men. A UK study demonstrated that males had significantly greater mortality than females [fully adjusted HR 1.5 (1.52-1.65)] [14]. This is likely due to the difference in research methods including sample size, population type, and definition of COVID-19 related mortality.

Hypertension, followed by heart disease was the most commonly encountered co-morbidities amongst the hospitalized COVID-19 patients in this study. Neither was associated with increased mortality, admission to ITU, or mechanical ventilation which is in keeping with recent literature [13,16]. An eGFR of $<60$

$\mathrm{mL} / \mathrm{min} / 1.73 \mathrm{~m}^{2}$ at the time of COVID-19 diagnosis was found in $44.1 \%$ of the patients (patients with chronic kidney disease [CKD] and acute kidney injury were both included), and this was associated with increased mortality. This supports recently published data that CKD and reduced admission eGFR were associated with increased mortality $[18,19]$. Angiotensin-converting enzyme 2 (ACE2) is thought to play an important role in acute kidney injury in patients with COVID-19. ACE2 is a membrane protein expressed in the kidneys, in addition to the lungs and heart [20]. The membrane spike protein on SARS-CoV-2 is thought to bind ACE2 receptors, permitting viral entry. The SARS-CoV-2 may infiltrate renal cells via the ACE2 receptor resulting in architectural damage. Furthermore, it has been proposed that COVID-19 has a direct cytotoxic effect on the kidneys [21].

\section{Prevalence of diabetes in patients with COVID-19}

Current evidence has demonstrated patients with COVID-19 and diabetes are more likely to develop severe COVID-19 [22]. 30.6\% of patients admitted to BSUH with COVID-19 had diabetes. Therefore, the prevalence of hospitalized patients with diabetes and COVID-19 was seven times greater than the local population 
prevalence of 4.2\% in Brighton and Hove, UK [23]. Alkundi et al. similarly reported a prevalence of 37.5\% [5]. However, our study had more patients with type 2 diabetes (96.4\%) when compared to Alkundi et al. (87.4\%) [5]. The number of patients with type 1 diabetes mellitus is too small to be representative of the population with type 1 diabetes. The current literature suggests that patients with type 1 diabetes mellitus are not at increased risk of hospitalization due to COVID-19 [24].

\section{Outcomes of COVID-19 in patients with diabetes}

Even though $29.4 \%$ of the total deaths were in patients with diabetes, there was no association with increased mortality. Patients with diabetes were significantly younger than those without diabetes, which may be why there was no difference in mortality between the two groups. Univariate analysis in the diabetes group showed that age was the most significant factor predicting mortality. However, multivariate analysis in the diabetes group could not be undertaken. There is conflicting data in the literature as to whether diabetes is associated with increased mortality in COVID-19 [5,25]. Although our results are in keeping with a single center UK-based study [5], they contrast a larger population-based study [8], which showed diabetes was associated with increased mortality. In some studies, poorer outcomes in patients with diabetes could be due to significantly older age in patients with diabetes than those without diabetes [15,25].

The proportion of patients in the $\leqslant 60$ and $>60$ years age groups was similar in diabetes and non-diabetes groups. All patients $\leqslant 60$ years old who died from COVID-19 had diabetes, although this represented only three patients. In patients $\leqslant 60$ years of age with diabetes, two out of three deaths occurred in the BAME population. However, regression analysis did not show BAME background was a risk factor for increased mortality. This is likely due to younger patients with a BAME background. There was no difference in mortality in patients with and without diabetes in $>60$ years of age.

It is well known that diabetes is associated with longer LoS in hospitalized patients [26]. We demonstrated that overall LoS from COVID-19 in our study was not significantly longer in the diabetes group. However, subgroup analysis demonstrated significantly more patients with diabetes stayed seven days or longer than those without diabetes, which may imply an increased COVID-19 disease severity and associated complications, hence requiring an extended admission. Furthermore, we demonstrated an increased LoS in patients with diabetes who were $\leqslant 60$ years. In contrast, patients aged $>60$ years did not show any difference in LoS. Current evidence with regards to COVID-19 and diabetes has been conflicting [15,27]. Wang et al. showed that there was no difference in LoS between patients with diabetes and those without diabetes [27]. In contrast, Chen et al. showed that patients with diabetes had a significantly longer LoS than those without diabetes [15].

\section{COVID-19 patients with diabetes and co-morbidities}

Our study showed that patients with diabetes had significantly more co-morbidities (HTN, heart disease, COPD, asthma, and renal impairment). However, these co-morbidities were not associated with increased mortality in patients with COVID-19 and diabetes. eGFR was associated with increased mortality in the overall analysis of all patients. Our data are supported by current evidence with regards to HTN, heart disease, and eGFR [13,19].

\section{Impact of glycemia in patients with COVID-19 and diabetes}

Poor glycaemic control in patients with COVID-19 has been associated with worse outcomes, including mortality [4]; however, we did not observe this within our cohort. This could be due to the differences in age between the patients with and without diabetes in our study compared to these studies. Patients with hyperglycaemic emergencies (DKA or/and HHS) did not have increased mortality. There are reports showing that patients with diabetes and COVID-19 are more likely to present with DKA and/or HHS [28]. It is known that patients with hyperglycaemic emergencies have increased mortality and morbidity [10,11]. In contrast, a recent study demonstrated that patients with COVID-19 and DKA had improved survival to those without DKA [5]. Further studies are needed to evaluate the effect of diabetic emergencies in patients with diabetes and COVID-19.

We demonstrated that the median blood glucose in patients with diabetes were acceptable. Hyperglycaemia has been reported with COVID-19 in patients with and without diabetes and is associated with worse outcomes, including increased mortality [16]. This is important in the context of the recent guidelines recommending administering dexamethasone in severe COVID-19 [29]. Therefore, an increased frequency of monitoring should be undertaken in patients with diabetes (in our study, around $28 \%$ of the patient had capillary blood glucose tested on $\leqslant 2$ occasions).

\section{Limitations}

Our study has some limitations. First, due to the retrospective study design, body mass index was not available, which is an important independent risk factor for mortality in COVID-19 [30]. Second, we did not include the duration of diabetes as this was not available in most of the records. Therefore, we could not ascertain if obesity and duration of diabetes are confounding variables affecting COVID-19 mortality. Third, we defined COVID-19-related death as patients dying within 28 days of a first positive laboratory-confirmed 
COVID-19 test. It may mean that we have under-calculated the number of deaths. In England, 88\% of the deaths reported up to August 3, 2020 occurred within 28 days of the first COVID-19 positive test. Death in patients who have tested positive for COVID-19 becomes progressively less likely to be as a result of COVID19 as the time from the diagnosis increases. Therefore, we based the mortality on Public Health England guidance [12]. In addition, as stated above, subgroup mortality analysis aged $\leqslant 60$ years included very few deaths, therefore, the result should be interpreted with caution. Our study is strengthened by only including patients who were SARS-CoV-2 PCR positive. We evaluated glycaemic control at the time of admission and during the hospital stay in patients with diabetes. Blood glucose levels at the time of admission may cause bias, particularly from reverse causality. Hyperglycemia has been associated with increasing severity of COVID-19 infection and poor prognosis for patients with or without diabetes [7]. Finally, all the data were obtained for patients during the first wave of the COVID-19 pandemic. The management of COVID-19 underwent significant changes including the use of Dexamethasone and Remdesivir. Some of the patients may have received these medications especially in the later cohort which could have influenced the outcome.

\section{Conclusions}

In conclusion, diabetes was only associated with increased mortality in patients $\leqslant 60$ years old. However, due to the small sample size, the results of the subgroup $\leqslant 60$ years old should be interpreted with caution. There was no association between glycaemic control, prior to and during hospitalization, and mortality. Patients with diabetes had longer hospital stays, which may imply greater disease severity. Older age was a significant risk factor for increased mortality in COVID-19. Prospective studies of patients with diabetes hospitalized with COVID-19 are required to determine the impact of glycemic emergencies and glycemic control on outcomes. Additional studies from other regions of the UK are needed to address whether there are regional differences in risks for mortality in patients with COVID-19.

\section{Additional Information \\ Disclosures}

Human subjects: Consent was obtained or waived by all participants in this study. Animal subjects: All authors have confirmed that this study did not involve animal subjects or tissue. Conflicts of interest: In compliance with the ICMJE uniform disclosure form, all authors declare the following: Payment/services info: All authors have declared that no financial support was received from any organization for the submitted work. Financial relationships: All authors have declared that they have no financial relationships at present or within the previous three years with any organizations that might have an interest in the submitted work. Other relationships: All authors have declared that there are no other relationships or activities that could appear to have influenced the submitted work.

\section{Acknowledgements}

Alisha Masani, Daisy Newberry, Tharaga Kirupakaran, Jenny Wilson, Jonathan Golding, Khaled Ghanem, B Naw Ra Aung Din, Jennifer Oseyi Eigbire, Ketan Patel contributed to the project by collecting the data.

\section{References}

1. WHO Director-General's opening remarks at the media briefing on COVID-19 - 11 March . (2020). Accessed: March 15, 2021: https://www.who.int/dg/speeches/detail/who-director-general-s-opening-remarks-at-themedia-briefing-on-covid-19---11-....

2. WHO Director-General's opening remarks at the media briefing on COVID-19-13 March . (2020). Accessed: March 15, 2021: https://www.who.int/director-general/speeches/detail/who-director-general-s-openingremarks-at-the-mission-briefing-o....

3. Dong E, Du H, Gardner L: An interactive web-based dashboard to track COVID-19 in real time . Lancet Infect Dis. 2020, 20:533-534. 10.1016/\$1473-3099(20)30120-1

4. Bode B, Garrett V, Messler J, McFarland R, Crowe J, Booth R, Klonoff DC: Glycemic characteristics and clinical outcomes of COVID-19 patients hospitalized in the United States. J Diabetes Sci Technol. 2020, 14:813-821. 10.1177/1932296820924469

5. Alkundi A, Mahmoud I, Musa A, Naveed S, Alshawwaf M: Clinical characteristics and outcomes of COVID19 hospitalized patients with diabetes in the United Kingdom: a retrospective single centre study. Diabetes Res Clin Pract. 2020, 165:108263. 10.1016/j.diabres.2020.108263

6. Huang I, Lim MA, Pranata R: Diabetes mellitus is associated with increased mortality and severity of disease in COVID-19 pneumonia - a systematic review, meta-analysis, and meta-regression. Diabetes Metab Syndr. 2020, 14:395-403. 10.1016/j.dsx.2020.04.018

7. Zhu L, She Z-G, Cheng X, et al.: Association of blood glucose control and outcomes in patients with COVID19 and pre-existing type 2 diabetes. Cell Metab. 2020, 31:1068-1077. 10.1016/j.cmet.2020.04.021

8. Barron E, Bakhai C, Kar P, et al.: Associations of type 1 and type 2 diabetes with COVID-19-related mortality in England: a whole-population study. Lancet Diabetes Endocrinol. 2020, 8:813-822. 10.1016/S22138587(20)30272-2

9. National Statistics: English indices of deprivation. (2019). Accessed: March 15, 2021: https://www.gov.uk/government/statistics/english-indices-of-deprivation-2019.

10. Joint British Diabetes Societies Inpatient Care Group. The management of diabetic ketoacidosis in adults . (2013). Accessed: March 15, 2021: 
https://abcd.care/sites/abcd.care/files/resources/2013_09_JBDS_IP_DKA_Adults_Revised.pdf.

11. Joint British Diabetes Societies Inpatient Care Group. The management of the hyperosmolar hyperglycaemic state (HHS) in adults with diabetes. (2012). Accessed: March 15, 2021:

https://abcd.care/sites/abcd.care/files/resources/JBDS_IP_HHS_Adults.pdf.

12. Public health matters. Behind the headlines: counting COVID-19 deaths . (2020). Accessed: March 15, 2021: https://publichealthmatters.blog.gov.uk/2020/08/12/behind-the-headlines-counting-covid-19-deaths/.

13. Iaccarino G, Grassi G, Borghi C, Ferri C, Salvetti M, Volpe M; SARS-RAS Investigators: Age and multimorbidity predict death among COVID-19 patients: results of the SARS-RAS study of the Italian Society of Hypertension. Hypertension. 2020, 76:366-372. 10.1161/hypertensionaha.120.15324

14. Williamson EJ, Walker AJ, Bhaskaran K, et al.: Factors associated with COVID-19-related death using OpenSAFELY. Nature. 2020, 584:430-436. 10.1038/s41586-020-2521-4

15. Chen Y, Yang D, Cheng B, et al.: Clinical characteristics and outcomes of patients with diabetes and COVID19 in association with glucose-lowering medication. Diabetes Care. 2020, 43:1399-1407. 10.2337/dc20-0660

16. Cariou B, Hadjadj S, Wargny M, et al.: Phenotypic characteristics and prognosis of inpatients with COVID19 and diabetes: the CORONADO study. Diabetologia. 2020, 63:1500-1515. 10.1007/s00125-020-05180-X

17. Suleyman G, Fadel RA, Malette KM, et al.: Clinical characteristics and morbidity associated with Coronavirus disease 2019 in a series of patients in metropolitan detroit. JAMA Netw Open. 2020, 3:e2012270. 10.1001/jamanetworkopen.2020.12270

18. Uribarri A, Núñez-Gil IJ, Aparisi A, et al.: Impact of renal function on admission in COVID-19 patients: an analysis of the international HOPE COVID-19 (Health Outcome Predictive Evaluation for COVID 19) Registry. J Nephrol. 2020, 33:737-745. 10.1007/s40620-020-00790-5

19. Zahid U, Ramachandran P, Spitalewitz S, et al.: Acute kidney injury in COVID-19 patients: an inner city hospital experience and policy implications. Am J Nephrol. 2020, 51:786-796. 10.1159/000511160

20. Jin Y, Yang H, Ji W, Wu W, Chen S, Zhang W, Duan G: Virology, epidemiology, pathogenesis, and control of COVID-19. Viruses. 2020, 12:372. 10.3390/v12040372

21. Pan F, Yang L, Li Y, et al.: Factors associated with death outcome in patients with severe coronavirus disease-19 (COVID- 19): a case-control study. Int J Med Sci. 2020, 17:1281-1292. 10.7150/ijms.46614

22. Apicella M, Campopiano MC, Mantuano M, Mazoni L, Coppelli A, Del Prato S: COVID-19 in people with diabetes: understanding the reasons for worse outcomes. Lancet Diabetes Endocrinol. 2020, 8:782-792. 10.1016/S2213-8587(20)30238-2

23. NHS Brighton and Hove CCG: CVD profiles - diabetes . (2020). Accessed: May 29, 2020: https://fingertips.phe.org.uk/reports/ssrs/? reportName=ncvin\%2FDiabetes_Profile\&areaCode=E38000021 \&areaTypeId=154\&par....

24. Vangoitsenhoven R, Martens P-J, van Nes F, et al.: No evidence of increased hospitalization rate for COVID19 in community-dwelling patients with type 1 diabetes. Diabetes Care. 2020, 43:118-119. 10.2337/dc201246

25. Yan Y, Yang Y, Wang F, et al.: Clinical characteristics and outcomes of patients with severe covid-19 with diabetes. BMJ Open Diabetes Res Care. 2020, 8:001343. 10.1136/bmjdrc-2020-001343

26. Malone M, Lau NS, White J, et al.: The effect of diabetes mellitus on costs and length of stay in patients with peripheral arterial disease undergoing vascular surgery. Eur J Vasc Endovasc Surg. 2014, 48:447-451. 10.1016/j.ejvs.2014.07.001

27. Wang X, Liu Z, Li J, et al.: Impacts of type 2 diabetes on disease severity, therapeutic effect, and mortality of patients with COVID-19. J Clin Endocrinol Metab. 2020, 105:535. 10.1210/clinem/dgaa535

28. Rafique S, Ahmed FW: A case of combined diabetic ketoacidosis and hyperosmolar hyperglycemic state in a patient with COVID-19. Cureus. 2020, 12:8965. 10.7759/cureus.8965

29. Group RC, Horby P, Lim WS, et al.: Dexamethasone in hospitalized patients with Covid-19. N Engl J Med. 2021, 384:693-704. 10.1056/NEJMoa2021436

30. Palaiodimos L, Kokkinidis DG, Li W, et al.: Severe obesity, increasing age and male sex are independently associated with worse in-hospital outcomes, and higher in-hospital mortality, in a cohort of patients with COVID-19 in the Bronx, New York. Metabolism. 2020, 108:154262. 10.1016/j.metabol.2020.154262 